\title{
Orthopedics Surgery Trainer with PPU-Accelerated Blood and Tissue Simulation
}

\author{
Wai-Man Pang ${ }^{1}$, Jing Qin ${ }^{1}$, Yim-Pan Chui ${ }^{1}$, Tien-Tsin Wong $^{1}$, \\ Kwok-Sui Leung ${ }^{2}$, and Pheng-Ann Heng ${ }^{1,3}$ \\ ${ }^{1}$ Dept. of Computer Science and Engineering, The Chinese University of Hong Kong \\ ${ }^{2}$ Department of Orthopaedics and Traumatology, CUHK \\ ${ }^{3}$ Shenzhen Institute of Advanced Integration Technology, \\ Chinese Academy of Science/CUHK
}

\begin{abstract}
This paper presents a novel orthopedics surgery training system with both the components for modeling as well as simulating the deformation and visualization in an efficient way. By employing techniques such as optimization, segmentation and center line extraction, the modeling of deformable model can be completed with minimal manual involvement. The novel trainer can simulate rigid body, soft tissue and blood with state-of-the-art techniques, so that convincing deformation and realistic bleeding can be achieved. More important, newly released Physics Processing Unit (PPU) is adopted to tackle the high requirement for physics related computations. Experiment shows that the acceleration gain from PPU is significant for maintaining interactive frame rate under a complex surgical environments of orthopedics surgery.
\end{abstract}

\section{Introduction}

Orthopedics surgery simulation is complex due to the co-existence of various kinds of body tissues with heterogeneous mechanical behaviors, such as bones, dermis, fatty tissues and blood vessels. This obviously makes the modeling and simulation more complicated and challenging.

Many existing simulators for Orthopedics surgeries are focusing on the interactivity and training procedures, while realism is compromised because of limited computational resources and the lack of realistic mechanical and anatomical data. For example, the physical simulation of bleeding and tissue deformation, which are indispensable components in orthopedics surgical simulation, are usually far from realistic. Besides, modeling of soft tissue and vessel are usually prepared manually, which may not reveal the correct anatomy and appearance of a real organ.

To meet these challenges, in this paper, we present a novel virtual reality (VR) system for Orthopedics surgery training. New consumer-level Physics Processing Unit (PPU) is used to accelerate the physical computation involved in bleeding simulation and tissue deformation. By exploiting the power of the PPU, high realism and interactive frame rate can be simultaneously achieved in a cost-effective way. Moreover, geometry construction and visualization of dermis, 
muscles and vessels are mainly based on anatomical information from Chinese Visible Human (CVH) dataset. This helps a lot in preparing a surgical environment similar to real situations. Finally, taumar surgery on upper limb is chosen as a particular application to demonstrate our system.

\section{Related Work}

Many academic and commercial VR-based orthopedics surgery simulators have been proposed for the training of surgeons 1 2 3. Most of them can be categorized into two classes based on the nature of surgery involved, namely arthroscopy and trauma surgery. McCarthy et al. 4] proposed the Sheffield Knee Arthroscopy Training System (SKATS), in which trainees are expected to learn the skill of navigating the knee arthroscopically. Haptic devices are incorporated to increase the learning speed of users. Reinig et al. 5] presented the US Militarys Thigh Trauma Simulator which provides a virtual environment with case scenarios of thigh trauma resulting in femur fractures. Our current system is mainly targeted towards trauma surgeries and tries to simulate the kind of open surgery in orthopedics involving incision.

Hardware acceleration for rendering has long been used in many graphics applications including surgical applications. Similar to rendering, physically based deformation is another essential and computationally intensive component in surgical simulations. Many researchers 67 have tried to exploit the power of the programmable GPU for physics computation, but they usually suffer from certain difficulties or limitations, such as the restriction on the topology of deformable model. Recently, a specialized hardware accelerator for physics known as Physical Processing Unit (PPU) is released for complex dynamic motion, interaction and physics computation [8]. More details of the capabilities of the PPU in our virtual orthopedics simulator will be introduced in section 4.

\section{Geometric Modeling}

In our system, creation of deformation and visualization models is based on anatomical information provided by the $\mathrm{CVH}$ dataset. In order for the models to resemble human organs, we employ a layered soft tissue model. Each layer is modeled as a mass-spring model with different topologies, like tetrahedron or lattice structures.

In the modeling of human dermal-muscular tissues, epidermis is in tetrahedron structure while others are in lattice structure as shown in Figure 1(a) and (b). Based on the segmented CVH, consistent polygonal surfaces of each tissue layer are extracted as Stereolithography (STL) format. By re-parameterizing the polygonal surface meshes into a quad-based surface, we can easily control the complexity of the surface and skip the manual smoothing procedures. Next, a multi-layered mass-spring model can be automatically produced based on the re-parameterized surface meshes. For the tetrahedron layer, every triangle in the upper surface projects its centroid in the normal direction to hit the lower 


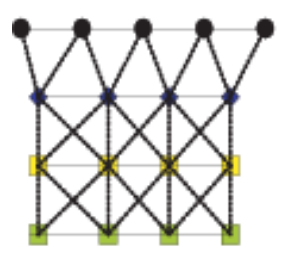

(a)

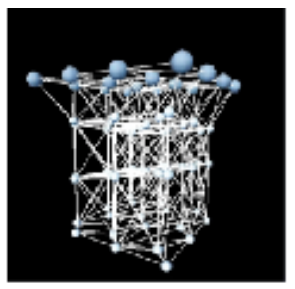

(b)

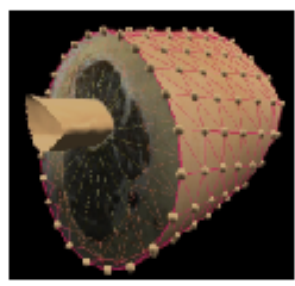

(c)

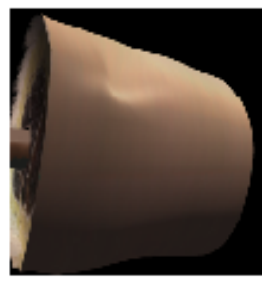

(d)

Fig. 1. The multilayer topology of mass-spring model, (a) a side view, (b) a 3D view, (c) the upper limb model from $\mathrm{CVH}$ data and (d) the deformed model by external pressure

surface layer. These intersection points, namely projected centroids, form the masses units in the lower layer. Then, springs are added to connect the vertices in original triangle and its projected centroid. This creates a tetrahedral mesh structure between the two surface layers. Afterwards, triangulation is carried out on these projected centroids. Similar processes are repeated for the lower layers until all layers are constructed.

Apart from the topology building of the mass-spring model, some auxiliary modules are used to extract boundary, texture from the CVH, adjust mass density and other mechanical properties. Figure 1(c) shows a textured mass-spring model generated using the CVH's upper limb.

To automatically construct the deformable vessel model, the centreline of the entire vascular structure have to be extracted first. Since bifurcations (branches) occur throughout the vascular network, a tree structure is deployed for storing this structure. After centerline extraction, we perform geometric modeling. First, a series of Bezier curves are fitted onto the extracted centreline where the distribution of control points depend on the local curvature of the vascular pathway. Bifurcations are handled through a modified Bezier triangle. Finally, the external surface structure are modeled through a swap of local frame.

\section{PPU-Accelerated Integrated Orthopedics Simulation}

Instead of concentrating on the simulation of bone,our system is a more comprehensive simulation system for different kinds of orthopedics surgery. Both realistic blood flow simulation and soft tissue deformation are supported in our integrated orthopedics simulator. These two components are most computationally intensive. Many of the previous simulators try to simplify their models for maintaining an interactive frame-rate, however, this will reduce the realism provided to the user. As a result, our system utilizes the recently released PPU to accelerate physics simulations. In addition to the deformation computation, the collision and interaction can be handled inside the PPU which only require construction of a collision mesh model at the initialization. 


\subsection{MSM-Based Soft Tissue Deformation}

It is well-known that softtissue exhibits non-linear viscoelasticity, that means the stress-strain relation is non-linear. In general, it has a very low stiffness at the beginning and extremely high stiffness after the tissue is stretched to a certain extent. Therefore, a biphasic mass-spring system is used to mimic the non-linear behavior of body tissues.

However, the latest PPU is built only with linear mass-spring system implementation, even though it allows for the definition of mass-spring in arbitrary topology. As a result, we need to extend the current linear mass-spring system by making use of two linear springs with different elastic configurations in parallel. The basic idea is letting the first linear spring be effective in the first phase of the stress-strain curve, while the second linear spring becomes activated when the turning point is reached. This simple construction of biphasic spring from linear springs leads to a simple decomposition of a biphasic spring into two linear springs and can be done automatically when the deformable model is initialized.

In order to obtain a macroscopic mass-spring system that is consistent with biomechanical properties of body tissue [9], we carry out an optimization process on (microscopic) individual spring elasticity. We choose simulated annealing for optimization, because it is very suitable for problems with many parameters and the first derivative of the objective function is unavailable. The objective function $\psi$ for optimization is formulated as Equation 1 .

$$
\psi=\sum_{i} w_{i}\left(p_{i}-q_{i}\right)^{2}
$$

where $p_{i}$ is the value of the $i$-th parameter from the experiment and $q_{i}$ is the corresponding value for that particular tissue coming from data books. $w_{i}$ is the weighting factor for that parameter. If biphasic spring is used, $i$ is 3 and $p_{i}$ are the first phase stiffness (K1), turning length and the second phase stiffness (K2); while for a linear spring, $i$ is 1 which referring to stiffness alone.

In order to evaluate $p_{i}$, experiments are carried out on the multilayer model repeatedly until a minimum objective is found. The springs in all tissue layers are optimized in a similar manner. Table 1 reports the mechanical properties of real dermis as well as our optimized model. Notice that the optimization process is only necessary to be performed once for each tissue. Figure 1(d) shows a deformed result of the upper limb model.

Theoretically, higher-order spring models should produce more realistic deformation, however, the visual difference is insignificant and the performance penalty induced by them might not worth. The relaxation of using high order spring is possible to certain tissue, for example, we did relax the spring to linear model like hypodermis.

Table 1. Comparison of real dermis elastic properties with our optimized result

\begin{tabular}{|c|c|c|c|}
\hline & $K_{1}(\mathrm{KPa})$ & $K_{2}(\mathrm{KPa})$ & Turning point (in strain) \\
\hline real dermis & 0.1 & 18.8 & 0.4 \\
\hline optimized model & 0.118 & 18.51 & 0.41 \\
\hline
\end{tabular}




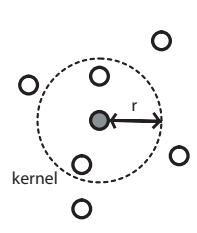

(a)

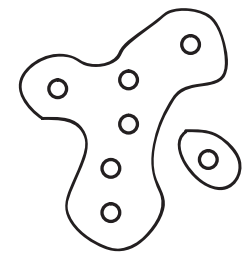

(b)

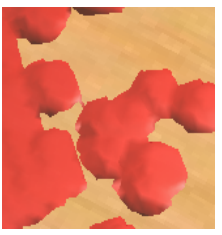

(c)

Fig. 2. The computational model of Smoothed Particle Hydrodynamics (SPH), (a) a particle and its inference kernel with radius $r$, (b) the blood surface extraction from particles, and (c) the rendered result

\subsection{SPH-Based Blood Flow Simulation}

Smoothed Particle Hydrodynamics (SPH) is known to be an efficient method in many graphics application for fluid animation 1011. Moreover, it can be accelerated with the PPU, so that sophisticated blood effects can run at a high frame rates.

$\mathrm{SPH}$ is a particle-based method for fluid simulation, in which nearby particles interact with each other according to the following formula,

$$
Q(l)=\sum_{j} a_{j} Q_{j} f\left(l-l_{j}, r\right)
$$

where $Q$ defines a scalar quantity at location $l$, which can be density, pressure and viscosity. It is a weighted sum of contributions from neighboring particles (denoted as $j$ ) within radius $r$, based on the field quantity $Q_{j}$ and inversely proportional to their distance $l-l_{j} \cdot a_{j}$ is equal to $\frac{m_{j}}{\rho_{j}}$, with $m_{j}$ and $\rho_{j}$ are the mass and density of particle $j$ respectively. The function $f(l, r)$ is referred as the smoothing kernel with radius $r$ as shown in Figure 2(a).

The governing equations for fluid dynamics are the Navier-Stokes equations, which formulate conservation of momentum as follow,

$$
\rho\left(\frac{\partial v}{\partial t}+v \cdot \nabla v\right)=-\nabla p+\rho g+\mu \nabla^{2} v
$$

Notice that the above formulations had assumed the fluid is newtonian (e.g. water). According to biomechanicalliteratures, blood is regarded as nonnewtonian fluid. However, by carefully adjusting the properties, we can achieve similar visual effect. In our experiments, we use $3 \mathrm{cP}$ for viscosity, $20 \mathrm{~N}$ for stiffness and $1.06 \mathrm{~g} / \mathrm{cm}^{-3}$ for density.

To render thesurface from a cloud of particles, there are many existing methods, such as level-set, marching cube or point splatting. For performance reasons, we use the marching cubes method to track the blood surface as a triangular mesh and then render it using the standard graphics pipeline.

$$
g=\nabla d
$$




$$
\begin{aligned}
\left|g\left(r_{i}\right)\right| & >\quad h \\
n & =-\frac{g\left(r_{i}\right)}{\left|g\left(r_{i}\right)\right|} .
\end{aligned}
$$

The surface tracking starts with the building of a color field $d$ within the space that the particles occupy. Then, we compute the gradient field $g$ using Equation 4 . surface elements are located using Equation 5 with $h$ being the threshold. Finally, the surface normal $n$ is obtained by normalizing $g$ according to Equation 6 .

\section{Time Performance and Visualization}

Experiments are carried out to compare the performance of a PPU-based system and a solely CPU-based system. Our two core components, the mass-spring modeled soft-tissue deformation and the SPH modeled bleeding simulation are used in the experiments. The test platform is a PC equipped with Pentium 4 Dual Core 3.2 GHz CPU and 4 GB memory, while the PPU is AGEIA PhysX Processor with $128 \mathrm{MB}$.

Figure 3(a) shows the experimental results of the mass-spring model. A significant speed improvement is observed in all grid-sizes with the use of the PPU, especially the double improvement in $15 \times 5$ and $18 \times 5$ grids. For fluid simulation using smoothed particles hydrodynamics method, the real-time frame rate is reported using the PPU when the number of particles is below 6,000 (see Figure 3(b)). With the same number of particles, pure CPU version decreases much faster in performance. The sudden performance drop at 6,000 particles in the PPU may be due to a data transfer bottleneck on the relatively slow PCI bus interface. From our experience, around 3,000 particles is enough for an acceptable bleeding effect. An integrated environment with both MSM $(39 \times 9$ mesh size) and SPH (5,000 particles) running can maintain an interactive frame rate of above 10 frames per second.

We demonstrate our orthopedics training system with an upper limb surgery training programme, in which a deformable upper limb is modeled as the

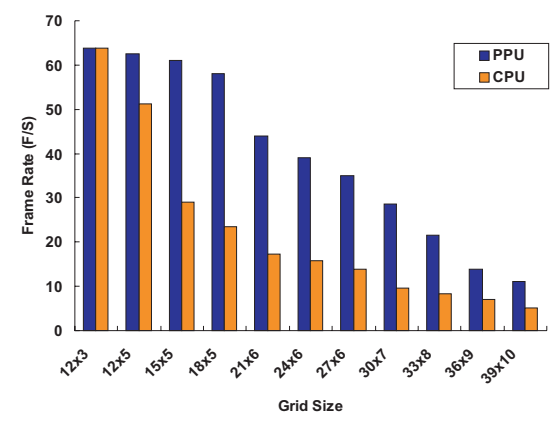

(a)

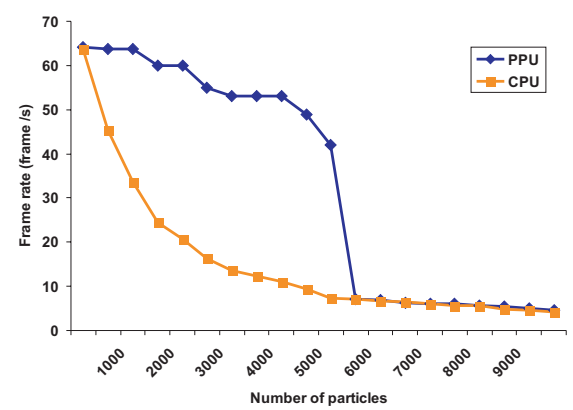

(b)

Fig. 3. Comparison on time performance of using the PPU and CPU for (a) massspring model deformation and (b) SPH bleeding simulation 


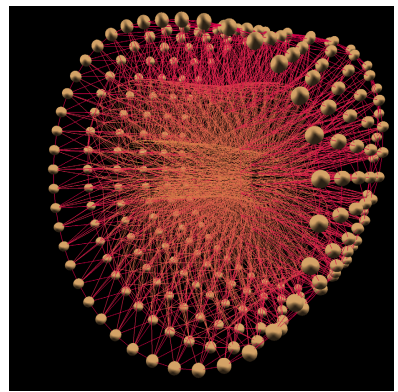

(a)

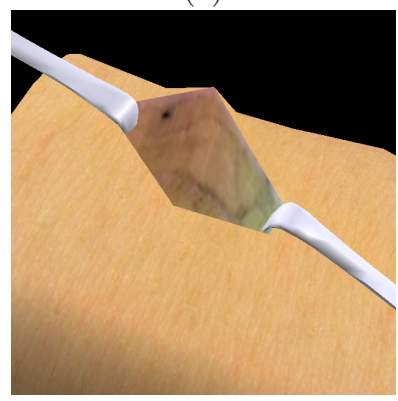

(d)

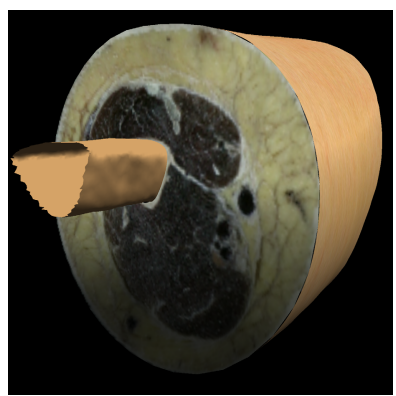

(b)

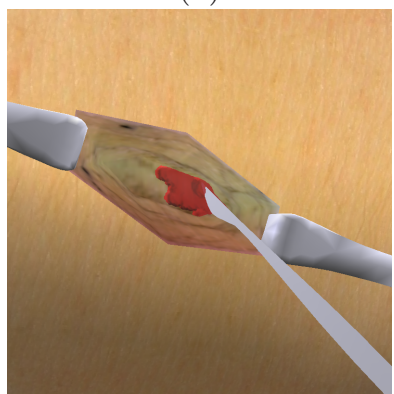

(e)

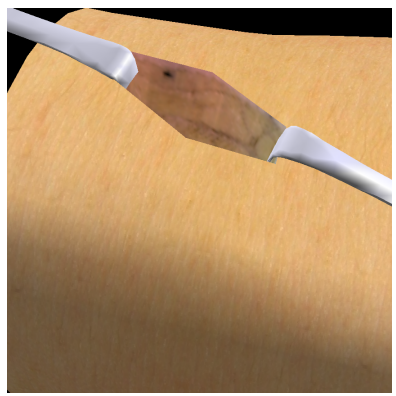

(c)

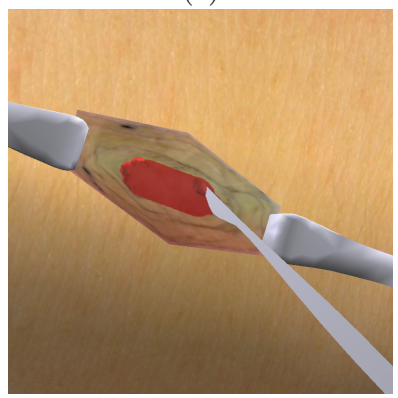

(f)

Fig. 4. Open orthopedic surgery: (a) the mass-spring model of the CVH upper limb, (b) the surface model, (c) the incision fixed by arms, (d) pulling on the incision, (e) an accidental cut at the blood vessel and (f) bleeding from the blood vessel

proposed mass-spring model and bleeding is simulated by the SPH particle method. By providing the generated mass-spring model, fluid particles and collision models to the PPU, we receive an updated coordinate of the masses and fluid particles in each time step. Based on these updated positions, we render the tissue models with texture from the CVH dataset. Figures 4 (a) and (b) show the mass-spring model and the surface model generated using an upper limb of the CVH dataset respectively. Figures 4(c) and (d) show an incision being done on the upper limb and that the limb can be deformed by a user for investigation during training. Bleeding is common during surgery, especially when there is accidental surgical fault, as shown in Figures 4(e) and (f). The user can practice handling bleeding using a draining tool. Realistic bleeding and soft-tissue deformation effects improve the experience given to the trainee.

\section{Conclusion and Future Work}

An integrated system for orthopedic surgery training is presented. The proposed method for modeling soft-tissue and blood by a combination of CVH dataset and biomechanics information is shown to be effective and efficient. By accelerating the computation with the PPU, a high fidelity of realism and interactive frame rate 
can be achieved in a virtual environment simulating both soft-tissue deformation and bleeding. Experiments have demonstrated the practical value of our work on an orthopedics trainer. We believe our work will be beneficial to similar surgical simulators as well. As a future work, we will try to enhance the visualization of blood and soft tissues by using more robust and advanced rendering techniques.

\section{Acknowledgments}

The work described in this paper was supported by a grant from the Research Grants Council of the Hong Kong Special Administrative Region (Project no. CUHK4223/04E). This work is also affiliated with the Virtual Reality, Visualization and Imaging Research Centre at The Chinese University of Hong Kong as well as the Microsoft-CUHK Joint Laboratory for Human-Centric Computing and Interface Technologies.

\section{References}

1. Olga, S., Alexei, S., Sen, H.T.: Orthopaedic surgery training simulation. Journal of Mechanics in Medicine and Biology, World Scientific (accepted, 2006)

2. Anderson, B.D., Nordquist, P., Skarman, E., Boies, M.T., Anderson, G.B., Carmack, D.B.: Integrated Lower Extremity Trauma Simulator. In: Westwood, J.D., et al. (eds.) Proceedings of Medicine Meets Virtual Reality, 2006, pp. 19-24. IOS Press, Amsterdam (2006)

3. Cannon, W.D., Eckhoff, D.G., Garrett, W.E., Hunter, R.E., Sweeney, H.J.: Report of a group developing a virtual reality simulator for arthroscopic surgery of the knee joint. Clinical Orthopaedics \& Related Research 442, 21-29 (2006)

4. McCarthy, A.D., Moody, L., Waterworth, A.R., Bickerstaff, D.R.: Passive haptics in a knee arthroscopy simulator: Is it valid for core skills training? Clinical Orthopaedics \& Related Research 442, 13-20 (2006)

5. Reinig, K., Lee, C., Rubinstein, D., Bagur, M., Spitzer, V.: The united states military's thigh trauma simulator. Clinical Orthopaedics \& Related Research 442, 45-56 (2006)

6. Georgii, J., Westermann, R.: Mass-spring systems on the gpu. Simulation Modelling Practice and Theory 13(8), 693-702 (2005)

7. Mosegaard, J., Sorensen, T.S.: A gpu accelerated spring-mass system for surgical simulation. In: MMVR 2005. Proceedings of 13th Medicine Meets Virtual Reality (MMVR), pp. 342-348 (2005)

8. Nealen, A., Muller, M., Keiser, R., Boxerman, E., Carlson, M.: Physically based deformablemodelsincomputergraphics. ComputerGraphicsForum25(4),809-836(2005)

9. Silver, F.H., Freeman, J.W., Devore, D.: Viscoelastic properties of human skin and processed dermis. Skin Research and Technology (7), 18-23 (2001)

10. Müller, M., Charypar, D., Gross, M.: Particle-based fluid simulation for interactive applications. In: SCA 2003. Proceedings of the 2003 ACM SIGGRAPH/Eurographics symposium on Computer animation, Aire-la-Ville, Switzerland, Switzerland, Eurographics Association, pp. 154-159 (2003)

11. Müller, M., Schirm, S., Teschner, M.: Interactive blood simulation for virtual surgery based on smoothed particle hydrodynamics. Technol. Health Care 12(1), 25-31 (2004) 